\title{
Land Evaluation of East Qattara Depression, Egypt using Remote Sensing and GIS
}

\author{
Hassan H. Abbas ${ }^{1}$, Ahmed S. Abuzaid ${ }^{1}$, Raffat K. Yaccub ${ }^{2}$ and Mostafa A. Mostafa ${ }^{2}$ \\ ${ }^{1}$ Soil and Water Department, Fac. of Agric., Benha Univ., Egypt \\ ${ }^{2}$ Soil, Water and Environ. Res. Inst. (SWERI), Agric. Res. Center (ARC), Egypt \\ Corresponding author: mostafa.adel.agr.egy@gmail.com
}

\begin{abstract}
Availability of sufficient and accurate spatial data concerning land resources is a pillar for sustainable agricultural development. The current work was aimed at using remote sensing (RS) and geostatistical analysis tools within geographic information system (GIS) to map land capability and crop suitability for an area in the Qattara Depressing, Western Desert of Egypt, located east of the Qattara Depression between latitude $30^{\circ} 10^{\prime} 4^{\prime \prime}$ to $30^{\circ} 20^{\prime} 57^{\prime \prime} \mathrm{N}$ and longitude $28^{\circ} 32^{\prime} 26^{\prime \prime}$ to $28^{\circ} 52^{\prime} 10^{\prime \prime} \mathrm{E}$, covering $630 \mathrm{~km}^{2}$ (63000 ha). Thirty-seven soil profiles were dug to $150 \mathrm{~cm}$. Soil samples were collected from different horizons and analyzed for their main properties. Applied System for Land Evaluation (ASLE) software was used to assess land capability and land suitability. Landforms include sand sheets, sand dunes, depressions, sabkha and water bodies. The $74.69 \%$ of the soils are"poor" (C4), $0.47 \%$ are"good" (C2), $11.68 \%$ are"fair" (C3), 11.12\% are"very poor" (C5), and 0.47\% are"non-agricultural" (C6). Soil texture, salinity and alkalinity are the main limiting factors. Classes for suitability (considering 22 crops) are highly suitable (S1), suitable (S2), moderately suitable (S3), marginally suitable (S4), currently not-suitable (N1), and potentially not-suitable (N2) for 22 crops. The most recommended crops are date palm and tomatoes. The studied soils require precise management practices to be promising for agricultural expansion.
\end{abstract}

Keywords: Remote sensing, GIS, Land capability, crop suitability, Qattara Depression

\section{Introduction}

The old fertile lands in the Nile Delta region of Egypt undergo various degradation processes that limited their potential exploitation for achieving food security. Therefore, great attention have been paid to the wide deserts that account for nearly $96 \%$ of the country total area (Abuzaid and Fadl, 2016). The Western Desert has gained more interest due to its unique nature such as flatness and presence of groundwater derived from the Nubian Sandstone Aquifer (Zahran and Willis, 2009). Qattara Depression is the largest and deepest megaDepressions of the Western Desert, and one of the world's greatest depressions. It has a total length from NE to SW ranging from 289 to $300 \mathrm{~km}$, width varying from 50 and $150 \mathrm{~km}$, and total surface area of 19605 $\mathrm{km}^{2}$ (Embabi, 2018)

Sustainable use of natural resources is one of the major objectives of the Egypt's Sustainable Agricultural Development Strategy (SADS) 2030 (Soliman, 2017). This requires a preciseevaluation to devote land resources for the optimum use (Mohamed et al., 2019). Land evaluation is the process of determining the fitness of land for a specific use based on its attributes and the ambient environmental conditions (Vasu et al., 2018). This process is an analysis integrating spatial information of various factors, including soil, climate, vegetation, topographic and hydrology, which is an analysis integrating spatial information (Xue, 2011). The results of this process should reach decision makers. Irrational use of land due to lack of proper awareness about land capability leads to destruction of such nonrenewable natural resource (Bacic et al., 2013).
Modern technologies; remote sensing (RS) and geographic information system (GIS) have been used increasingly in the field of land resources assessment (Ismail et al., 2013; Abuzaid and Fadl, 2016). Over the conventional methods, such technologies are effective tools in acquiring data for agricultural planning(Reddy, 2018b). This is because studies related to field work and natural resources depend mainly upon on the availability of sufficient and accurate spatial data, i.e. topography, water resources, land use, infrastructure, climate, land cover, geology, and manufacturing infrastructure (Sakai, 2012; Reddy, 2018a). Integrating spatial and non-spatial data of land resources in addition to their combined analysis can be carried out effectively under GIS environment. This enables an accurate inventory, mapping, monitoring and management of natural resources that help decision makers in sustainable agricultural planning (Reddy et al., 2018).

The main objective of the current work was utilizing RS and GIS for mapping land capability and crop suitability of soils located in the Qattara Depression. Such work would help decision makers in drawing a sustainable agricultural planning in this important region in the Western Desert of Egypt.

\section{Materials and methods}

\section{Study area}

The study area is located in the eastern part of Qattara Depression (Fig. 1) between latitude $30^{\circ} 10^{\prime}$ $4^{\prime \prime}$ to $30^{\circ} 20^{\prime} 57^{\prime \prime} \mathrm{N}$ and longitude $28^{\circ} 32^{\prime} 26^{\prime \prime}$ to $28^{\circ}$ $52^{\prime} 10^{\prime \prime} \mathrm{E}$, covering an area of $630 \mathrm{~km}^{2}$. The minimum temperature is $6.0{ }^{\circ} \mathrm{C}$ (in January), while the maximum one is $36.5^{\circ} \mathrm{C}$ (in July). Themean annual 
temperature of $20.94{ }^{\circ} \mathrm{C}$ and the total annual rainfall is $13.0 \mathrm{~mm}$. This indicatesa "Thermic" soil temperature regime and "Torric" soil moisture regime.

\section{RS and GIS works}

One scene with T35RPP title number and $10 \mathrm{~m}$ spatial resolution of Sentinel-2A Multispectral Instrument (MSI) sensor satellite covering the studied area was acquired from the Copernicus Data Hub gateway (https://scihub.copernicus.eu/) on 5/5/2016 (Fig. 2). A digital image processing (radiometric correction, spatial enhancement and classification) was done using ERDAS IMAGINE 2014 software. Topographic map sheets with a scale of 1:50000
(Egyptian General Survey Authority) were scanned and georeferenced (UTM projection, Zone 35 and Datum WGS-84) within ArcGIS 10.4 software (ESRI Co, Redlands, USA). Contour lines in every $0.5 \mathrm{~m}$ intervals and spot heights were digitized. A digital elevation model (DEM) covering the area was generated using geostatistical analysis. Geological features were imported from scanned and georeferenced geological maps with a scale of 1:50000 (Egyptian Geological Survey and Mining Authority). Based on the classified Sentinel-2A image, DEM and geological map, the geomorphic units of the studied area were extracted according to Zinck and Valenzuela (1990).

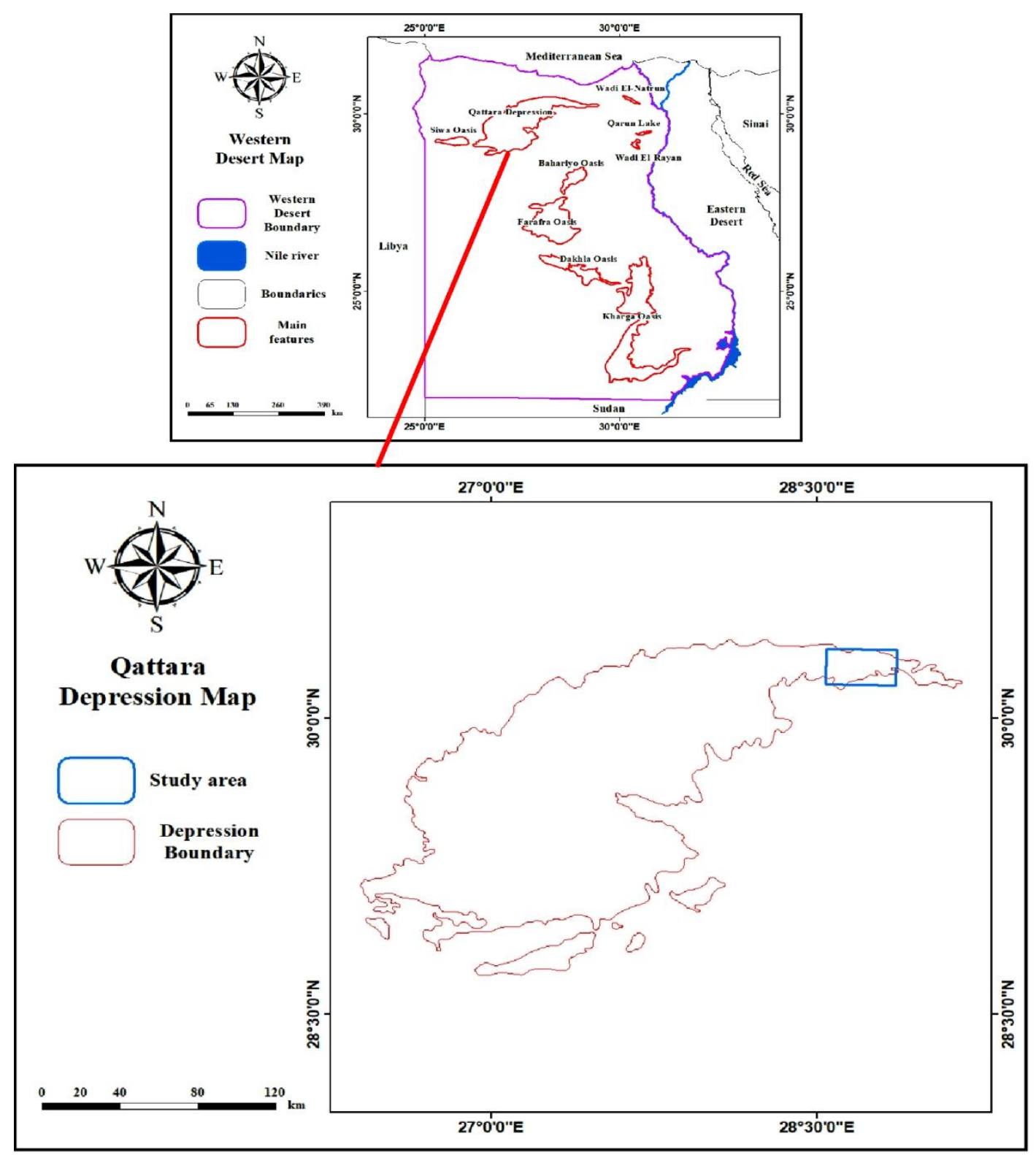

Fig. 1. Location map if the study area 


\section{Field work and laboratory analysis}

Thirty-seven soil profiles were dug to a $150 \mathrm{~cm}$ depth, unless opposed by rock formations or permanent water table. The Global Positioning System (GPS) was used to define the exact locations of soil profiles (Fig. 2). A detailed description of soil profiles were done according to (FAO, 2006). Soil samples (102 samples) were collected from the profiles to represent the subsequence horizons of each profile and kept for different laboratory analysis. Soil samples were air dried, crushed, and passed through a 2-mm sieve. Soil physicochemical analyses were performed according standard procedures (Soil Survey Staff, 2014).

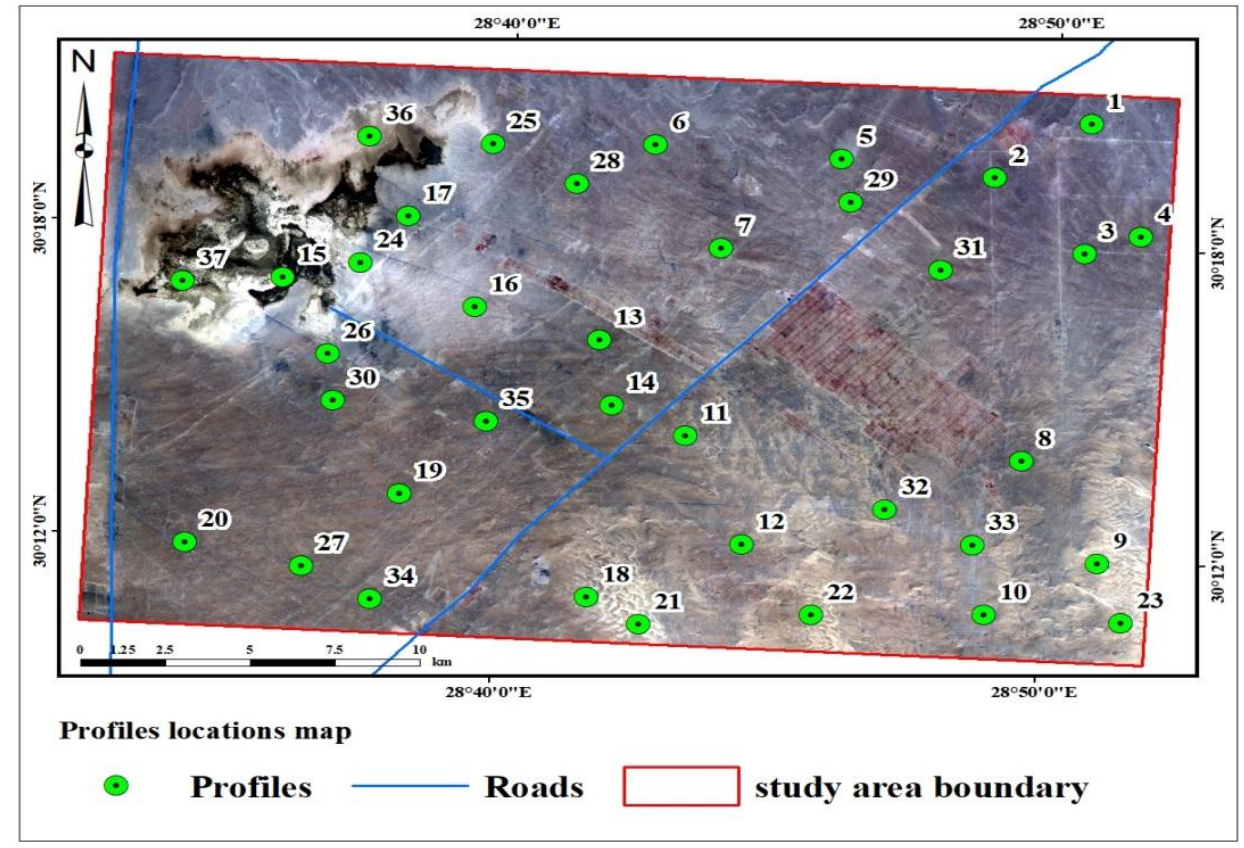

Fig. 1. Sentinel-2A image of the study area and profile locations

\section{Land evaluation}

The Applied System for Land Evaluation (ASLE) software (Ismail and Morsy, 2001) was used to predict land capability and crop suitability in the studied area. Land suitability based on matching crop requirements proposed by Sys et al. (1993) with soil properties. The factors reverent to the study are clay content, profile depth, land form, level of surface and slope, $\mathrm{pH}$, $\mathrm{CaCO}_{3}$, gypsum, cation exchange capacity (CEC), exchangeable sodium percentage (ESP) and salinity (expressed as EC). Capability and suitability classes are shown in Table 1.

\section{Generating capability and suitability maps}

The geostatistical analysis using the inverse distance weighting (IDW) technique was used for this purpose. The IDW predicts unknown values; (v) for any geographic point (x) using a number (n) and weight (w) of measured surrounding sample points (m). The equation is as as follows:

$$
\mathrm{v}(\mathrm{x})=\sum_{\mathrm{i}=1}^{\mathrm{n}} \mathrm{w}_{\mathrm{i}} \mathrm{m}_{\mathrm{i}} / \sum_{\mathrm{i}=1}^{\mathrm{n}} \mathrm{w}_{\mathrm{i}}
$$

The weight is calculated using the distance (d) between the point $\mathrm{x}$ and the neighbor point $\mathrm{m}$ and the power parameter (p), which determines the significance of sample points upon the interpolated value as follows:

$$
\mathrm{w}_{\mathrm{i}}=1 / \mathrm{d}_{\mathrm{i}}^{\mathrm{p}}
$$

Table 1. Land capability and suitability indices and classes of the ASLE

\begin{tabular}{llllll}
\hline $\begin{array}{l}\text { Capability } \\
\text { index (CI) }\end{array}$ & Class & Description & $\begin{array}{l}\text { Suitability } \\
\text { index (SI) }\end{array}$ & Class & Description \\
\hline$>80$ & C1 & Excellent & $>80$ & S1 & Highly suitable \\
$80-60$ & C2 & Good & $80-60$ & S2 & Suitable \\
$60-40$ & C3 & Fair & $60-40$ & S3 & Moderately suitable \\
$40-20$ & C4 & Poor & $40-20$ & S4 & Marginally suitable \\
$20-10$ & C5 & Very poor & $20-10$ & Ns1 & Currently not suitable \\
$<10$ & C6 & Non-agriculture & $<10$ & Ns2 & Permanently not suitable \\
\hline
\end{tabular}




\section{Results and discussion}

\section{Geomorphology}

The studied area includes five landforms; sand sheets (relatively high and relatively low), sand dunes (longitudinal and pyramid), depressions, sabkha and water bodies (Fig. 3). The sand sheets cover over three-quarters of the area $\left(74.96 \% ; 472.22 \mathrm{~km}^{2}\right)$ and include two units; relatively high $\left(238.42 \mathrm{~km}^{2}\right)$ and relatively low $\left(233.80 \mathrm{~km}^{2}\right)$. The sand dunes are located in the southern parts covering an area of 30.57 $\mathrm{km}^{2}$ (4.85\% of the total area). Two types of sand dunes are presented; longitudinal sand dunes with an area of $27.34 \mathrm{~km}^{2}$ and pyramid sand dunes with an area of 3.32 $\mathrm{km}^{2}$. Depressionsoccurin the form of small scattered areas covering $76.89 \mathrm{~km}^{2}$ (12.20\% of the total area). The sabkha is located in the northwestern part with an area of $36.09 \mathrm{~km}^{2}$, representing $5.73 \%$ of the total area.Water bodies, in the form of shallow water lakes surrounding sabkha deposits, occupy $14.23 \mathrm{~km}^{2}$, i.e. $2.26 \%$ of the total area.

\section{Soil properties}

Soil depth varies from 120 to $150 \mathrm{~cm}$, indicating deep to very deep soils (Table 2). According to Soil Science Division Staff (2017), the soils are moderately to strongly alkaline with a $\mathrm{pH}$ range of 7.35 to 8.69 , and none-saline to strongly saline with an EC ranging from 0.40 to $118.80 \mathrm{dS} \mathrm{m}^{-1}$. Soil organic matter content is very low (Hazelton and Murphy, 2016) with a range of 0.01 to $3.10 \mathrm{~g} \mathrm{~kg}^{-1}$. Soils gypsum content is $<100 \mathrm{~g} \mathrm{~kg}^{-1}$, thus it considered low (FAO, 1990). The soils $\mathrm{CaCO}_{3}$ ranges from 2.60 to $309.30 \mathrm{~g} \mathrm{~kg}^{-1}$. The CEC ranges from low to very high (Hazelton and Murphy, 2016) with a range of 6.78 to 49.19 comlc $\mathrm{kg}^{-1}$. The ESP ranges from 3.48 to 60.66 , which indicates none to very high sodicity (alkalinity) hazards(FAO, 1988). The sand fraction dominates soil particles averaging $85.61 \%$ followed by silt $(9.55 \%)$ and clay $(4.86 \%)$. Soil texture varies from sand to clay with sand being the most dominant textural class.

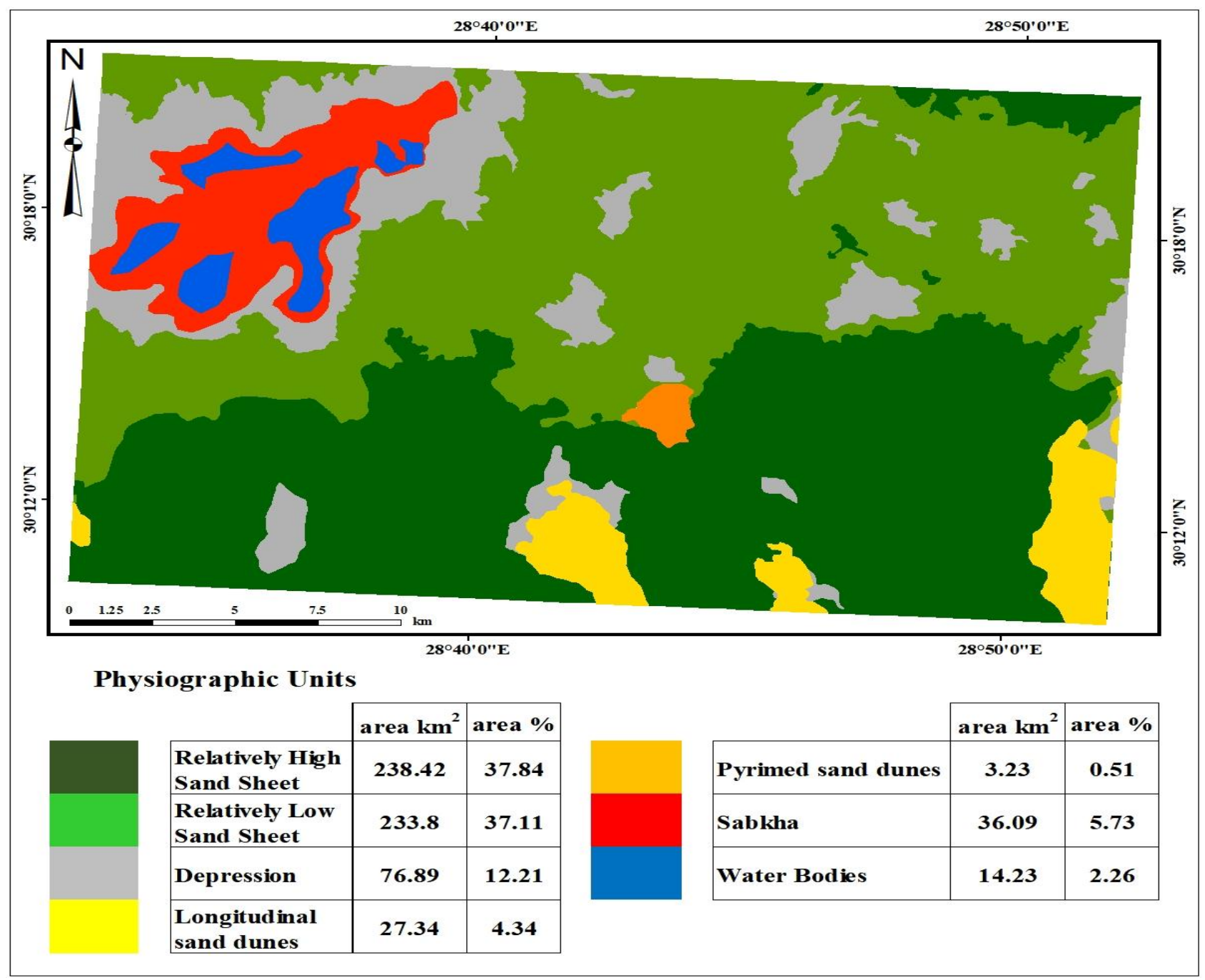

Fig. 3. Geomorphic map of the studied area 


\section{Land capability classification}

The spatial distribution of land capability classes (Fig. 4) demonstrates that $74.96 \%$ of the studies soils are poor $(\mathrm{C} 4)$, while the remaining area is occupied by good (C2), fair (C3), very poor (C5) and nonagricultural soils (C6). These classes represent 0.47 , $11.68,11.12$ and $1.77 \%$ of the total soils, respectively. The main limiting factors are soil texture, salinity and sodicity (alkalinity). Sandy soils have inherent poor fertility status and low water-holding capacity(Bassouny and Abuzaid, 2017). High clay content results in unfavorable physical conditions that cause adverse effects on plant growth and development. Unfavorable conditions include low infiltration rate, poor drainage, and poor aeration (Hazelton and Murphy, 2016). Salinity and alkalinity are two processes dominating arid and semi-arid soils and induce a major abiotic stress that limits plant productivity(Jafari et al., 2018).

Table 2. Soil physicochemical properties of the studied area

\begin{tabular}{|c|c|c|c|c|c|c|c|c|c|c|}
\hline Unit & $\begin{array}{l}\text { Profil } \\
\text { e No. }\end{array}$ & $\begin{array}{l}\text { Depth, } \\
\mathrm{cm}\end{array}$ & $\mathrm{pH}^{*}$ & $\begin{array}{l}\mathrm{EC}, * * \\
\mathrm{dS} \mathrm{m}^{-1}\end{array}$ & $\underset{\mathrm{kg}^{-1}}{\mathrm{OM}, \mathrm{g}}$ & $\begin{array}{c}\mathrm{CaCO}_{3} \\
\mathrm{~g} \mathrm{~kg}^{-1}\end{array}$ & $\begin{array}{c}\text { Gypsu } \\
\mathrm{m}, \\
\mathrm{g} \mathrm{kg}^{-1}\end{array}$ & $\begin{array}{c}\text { CEC, } \\
\text { cmolc } \\
\mathrm{kg}^{-1} \\
\end{array}$ & ESP & $\begin{array}{l}\text { Soil } \\
\text { texture }\end{array}$ \\
\hline \multirow{28}{*}{ 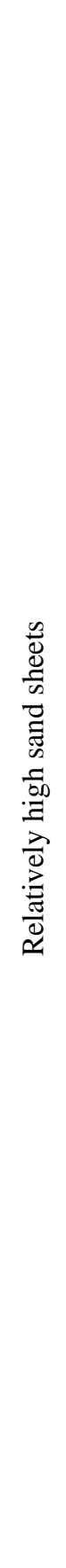 } & \multirow{3}{*}{1} & $0-35$ & 8.31 & 0.79 & 2.20 & 117.00 & 9.50 & 7.60 & 4.70 & $\begin{array}{c}\text { Loamy } \\
\text { sand }\end{array}$ \\
\hline & & $35-70$ & 8.24 & 3.24 & 0.80 & 69.00 & 0.40 & 7.65 & 7.73 & $\begin{array}{c}\text { Loamy } \\
\text { sand }\end{array}$ \\
\hline & & $70-150$ & 8.21 & 13.76 & 2.10 & 20.00 & 4.90 & 8.18 & 11.95 & Sand \\
\hline & \multirow{3}{*}{8} & $0-30$ & 7.78 & 0.96 & 1.50 & 23.00 & 4.30 & 7.64 & 4.80 & $\begin{array}{c}\text { Loamy } \\
\text { sand }\end{array}$ \\
\hline & & $30-70$ & 7.73 & 0.64 & 1.30 & 11.10 & 0.10 & 8.18 & 5.38 & Sand \\
\hline & & $70-150$ & 7.79 & 1.56 & 1.00 & 10.20 & 2.40 & 8.64 & 6.51 & Sand \\
\hline & \multirow{3}{*}{10} & $0-30$ & 8.08 & 1.44 & 0.90 & 5.90 & 3.90 & 8.18 & 5.85 & Sand \\
\hline & & $30-80$ & 7.92 & 6.56 & 0.40 & 5.90 & 12.00 & 8.09 & 8.52 & Sand \\
\hline & & $80-150$ & 7.95 & 7.60 & 1.90 & 5.70 & 2.80 & 8.64 & 8.93 & Sand \\
\hline & \multirow{3}{*}{12} & $0-20$ & 8.06 & 2.30 & 1.40 & 31.50 & 5.90 & 7.36 & 6.71 & $\begin{array}{c}\text { Loamy } \\
\text { sand }\end{array}$ \\
\hline & & $20-50$ & 8.16 & 1.60 & 2.00 & 10.80 & 1.40 & 8.30 & 10.13 & Sand \\
\hline & & $50-150$ & 8.11 & 19.00 & 1.80 & 11.50 & 1.90 & 8.41 & 9.48 & Sand \\
\hline & \multirow{3}{*}{19} & $0-30$ & 8.19 & 1.33 & 0.40 & 50.80 & 5.10 & 8.30 & 4.32 & Sand \\
\hline & & $30-60$ & 8.25 & 1.02 & 2.00 & 31.50 & 13.10 & 8.29 & 4.98 & Sand \\
\hline & & $60-150$ & 7.55 & 8.92 & 0.70 & 23.10 & 13.20 & 8.37 & 12.47 & Sand \\
\hline & \multirow{3}{*}{20} & $0-30$ & 7.70 & 4.33 & 0.40 & 30.80 & 3.20 & 8.63 & 18.22 & Sand \\
\hline & & $30-70$ & 7.90 & 24.30 & 2.30 & 11.50 & 12.40 & 8.64 & 36.10 & Sand \\
\hline & & $70-150$ & 7.54 & 19.45 & 1.00 & 10.00 & 3.90 & 8.64 & 16.03 & Sand \\
\hline & \multirow{3}{*}{32} & $0-30$ & 8.10 & 0.58 & 1.40 & 10.70 & 21.50 & 8.56 & 5.20 & Sand \\
\hline & & $30-65$ & 7.93 & 0.87 & 0.20 & 11.60 & 29.90 & 8.55 & 5.66 & Sand \\
\hline & & $65-125$ & 8.03 & 0.55 & 2.40 & 14.30 & 28.30 & 8.63 & 4.84 & Sand \\
\hline & \multirow{3}{*}{ S } & $0-25$ & 7.66 & 1.28 & 2.70 & 13.70 & 31.60 & 7.10 & 9.84 & $\begin{array}{c}\text { Loamy } \\
\text { sand }\end{array}$ \\
\hline & & $25-65$ & 7.72 & 1.06 & 0.40 & 12.30 & 38.20 & 8.56 & 10.79 & Sand \\
\hline & & $65-130$ & 7.67 & 1.23 & 0.70 & 17.80 & 26.90 & 8.68 & 9.83 & Sand \\
\hline & \multirow{3}{*}{34} & $0-35$ & 7.76 & 1.29 & 1.50 & 6.90 & 15.90 & 6.88 & 5.73 & $\begin{array}{c}\text { Loamy } \\
\text { sand }\end{array}$ \\
\hline & & $35-65$ & 7.70 & 0.98 & 1.00 & 10.30 & 53.80 & 8.81 & 6.39 & Sand \\
\hline & & $65-130$ & 7.71 & 1.19 & 1.30 & 14.40 & 33.50 & 8.81 & 4.26 & Sand \\
\hline & 35 & $0-35$ & 7.99 & 0.73 & 0.90 & 9.90 & 36.90 & 8.40 & 4.59 & Sand \\
\hline
\end{tabular}




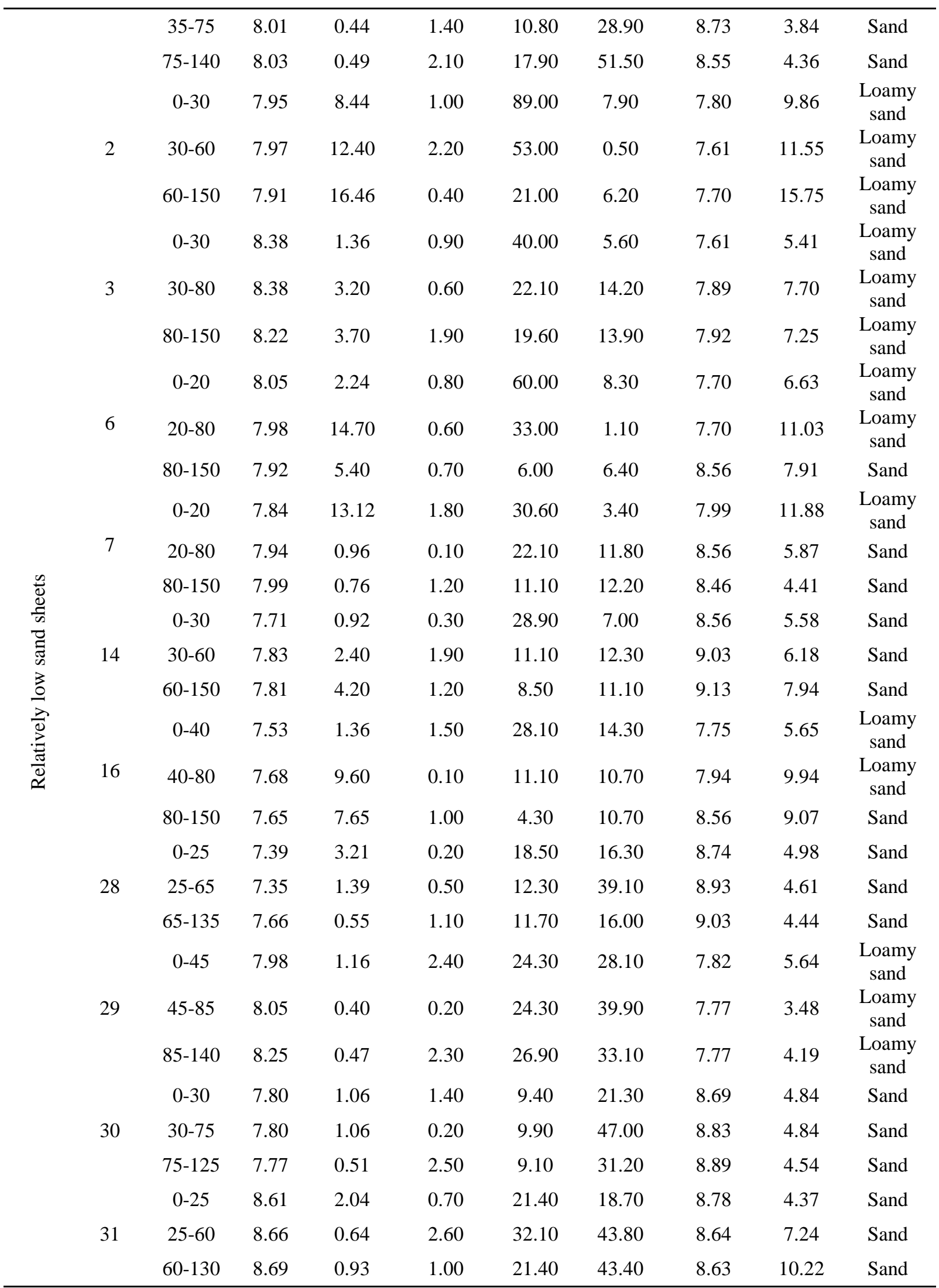

EC, electrical conductivity; * 1:2.5 soil : water suspension; ** soil paste extract; OM, organic matter; CEC, cation exchange capacity; ESP, exchangeable sodium percentage 
Table 2. Cont.

\begin{tabular}{|c|c|c|c|c|c|c|c|c|c|c|}
\hline Unit & $\begin{array}{c}\text { Profile } \\
\text { No. }\end{array}$ & $\begin{array}{c}\text { Depth, } \\
\text { cm }\end{array}$ & $\mathrm{pH}$ & $\begin{array}{c}\mathrm{EC} \\
\mathrm{dS} \mathrm{m} \mathrm{m}^{-1}\end{array}$ & $\begin{array}{l}\mathrm{OM}, \\
\mathrm{g} \mathrm{kg}^{-1}\end{array}$ & $\begin{array}{c}\mathrm{CaCO}_{3} \\
\mathrm{~g} \mathrm{~kg}^{-1} \\
\end{array}$ & $\begin{array}{c}\text { Gypsu } \\
\mathrm{m}, \\
\mathrm{g} \mathrm{kg}^{-1}\end{array}$ & $\begin{array}{c}\mathrm{CEC}, \\
\mathrm{cmolc} \\
\mathrm{kg}^{-1}\end{array}$ & ESP & $\begin{array}{c}\text { Soil } \\
\text { texture }\end{array}$ \\
\hline \multirow{10}{*}{ 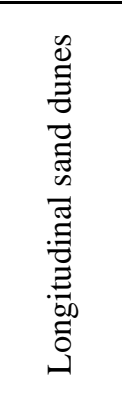 } & \multirow{2}{*}{9} & $0-40$ & 8.01 & 2.06 & 1.20 & 18.70 & 10.70 & 9.67 & 5.98 & Sand \\
\hline & & $40-150$ & 8.18 & 1.36 & 2.40 & 11.10 & 8.70 & 9.77 & 5.41 & Sand \\
\hline & \multirow{2}{*}{18} & $0-50$ & 8.06 & 0.48 & 1.90 & 11.90 & 4.00 & 9.46 & 3.96 & Sand \\
\hline & & $50-150$ & 8.06 & 3.04 & 0.10 & 5.10 & 1.00 & 9.98 & 7.55 & Sand \\
\hline & \multirow{2}{*}{21} & $0-40$ & 7.91 & 2.20 & 2.18 & 10.70 & 11.90 & 9.80 & 10.24 & Sand \\
\hline & & $40-120$ & 7.82 & 2.57 & 2.81 & 17.80 & 2.90 & 9.60 & 14.29 & Sand \\
\hline & \multirow{2}{*}{22} & $0-45$ & 8.00 & 0.62 & 0.20 & 3.70 & 3.50 & 9.46 & 4.25 & Sand \\
\hline & & $45-150$ & 8.07 & 2.15 & 2.60 & 3.70 & 12.90 & 9.67 & 6.89 & Sand \\
\hline & \multirow{2}{*}{23} & $0-50$ & 7.39 & 3.00 & 2.00 & 14.40 & 4.30 & 10.03 & 13.05 & Sand \\
\hline & & $50-150$ & 7.44 & 2.24 & 0.80 & 14.40 & 6.00 & 10.38 & 9.05 & Sand \\
\hline \multirow{12}{*}{$\begin{array}{c}\text { Pyrami } \\
\text { d }\end{array}$} & \multirow{2}{*}{11} & $0-30$ & 8.30 & 1.20 & 0.50 & 45.00 & 12.30 & 9.98 & 5.39 & Sand \\
\hline & & $30-150$ & 8.15 & 1.56 & 0.40 & 25.00 & 3.50 & 9.46 & 6.51 & Sand \\
\hline & \multirow{4}{*}{4} & $0-40$ & 8.12 & 4.72 & 0.01 & 2.60 & 0.50 & 7.72 & 8.96 & $\begin{array}{c}\text { Sandy } \\
\text { loam }\end{array}$ \\
\hline & & $40-80$ & 8.16 & 3.88 & 1.50 & 14.50 & 12.70 & 9.82 & 8.38 & Sand \\
\hline & & $80-150$ & 8.04 & 1.40 & 0.20 & 4.70 & 14.00 & 9.87 & 5.63 & Sand \\
\hline & & $0-30$ & 7.74 & 17.44 & 0.40 & 56.00 & 13.70 & 9.77 & 12.93 & Sand \\
\hline & \multirow[t]{2}{*}{5} & $30-80$ & 7.79 & 13.76 & 0.70 & 61.00 & 12.50 & 9.77 & 11.95 & Sand \\
\hline & & $80-150$ & 7.82 & 31.20 & 0.10 & 30.00 & 4.00 & 9.77 & 15.76 & Sand \\
\hline & \multirow{4}{*}{13} & $0-30$ & 7.58 & 3.20 & 0.40 & 19.60 & 5.10 & 6.78 & 7.59 & $\begin{array}{c}\text { Sandy } \\
\text { loam }\end{array}$ \\
\hline & & $30-80$ & 7.69 & 2.52 & 2.10 & 11.10 & 6.00 & 9.98 & 6.67 & Sand \\
\hline & & $80-150$ & 7.73 & 0.96 & 0.40 & 4.30 & 13.50 & 10.09 & 5.87 & Sand \\
\hline & & $0-30$ & 7.91 & 19.24 & 0.70 & 59.50 & 4.40 & 9.82 & 14.08 & Sand \\
\hline \multirow{16}{*}{ 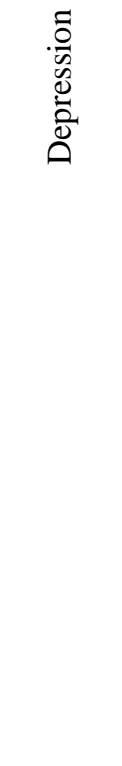 } & \multirow[t]{3}{*}{17} & $30-80$ & 7.95 & 51.52 & 1.40 & 57.70 & 1.40 & 9.66 & 20.78 & Sand \\
\hline & & $80-150$ & 7.96 & 48.94 & 2.20 & 22.00 & 5.90 & 9.55 & 19.74 & Sand \\
\hline & & $0-30$ & 7.90 & 1.09 & 1.50 & 51.50 & 14.60 & 9.77 & 5.15 & Sand \\
\hline & \multirow[t]{3}{*}{24} & $30-70$ & 7.88 & 4.08 & 0.80 & 53.60 & 18.20 & 7.70 & 8.30 & $\begin{array}{c}\text { Sandy } \\
\text { loam }\end{array}$ \\
\hline & & $70-125$ & 7.81 & 17.58 & 0.50 & 53.60 & 13.70 & 7.65 & 14.21 & $\begin{array}{c}\text { Sandy } \\
\text { loam }\end{array}$ \\
\hline & & $0-30$ & 7.87 & 0.76 & 0.30 & 35.90 & 10.00 & 10.03 & 4.86 & Sand \\
\hline & \multirow[t]{3}{*}{25} & $30-65$ & 7.89 & 13.10 & 0.60 & 26.90 & 39.10 & 10.33 & 11.99 & Sand \\
\hline & & $65-130$ & 7.95 & 13.92 & 0.20 & 33.90 & 50.10 & 9.91 & 12.28 & Sand \\
\hline & & $0-30$ & 7.66 & 1.59 & 1.90 & 12.30 & 10.90 & 10.50 & 5.12 & Sand \\
\hline & \multirow[t]{3}{*}{26} & $30-60$ & 7.63 & 2.55 & 1.00 & 9.60 & 10.50 & 10.39 & 14.04 & Sand \\
\hline & & $60-120$ & 7.43 & 4.60 & 0.70 & 9.60 & 7.80 & 10.61 & 13.51 & Sand \\
\hline & & $0-25$ & 7.87 & 0.88 & 0.30 & 17.20 & 14.60 & 10.58 & 4.30 & Sand \\
\hline & \multirow[t]{2}{*}{27} & $25-70$ & 7.44 & 4.20 & 0.70 & 9.60 & 40.00 & 10.72 & 16.17 & Sand \\
\hline & & $70-120$ & 7.55 & 4.62 & 0.50 & 10.30 & 41.90 & 10.69 & 27.91 & Sand \\
\hline & \multirow{2}{*}{15} & $0-35$ & 8.30 & 97.74 & 2.10 & 69.00 & 21.80 & 17.88 & 57.96 & $\begin{array}{l}\text { Silt } \\
\text { loam }\end{array}$ \\
\hline & & $35-150$ & 8.50 & 118.80 & 1.50 & 256.50 & 62.70 & 44.00 & 60.66 & Clay \\
\hline \multirow{4}{*}{ 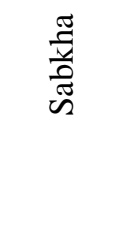 } & \multirow{2}{*}{36} & $0-40$ & 8.30 & 87.58 & 1.30 & 44.40 & 18.20 & 20.79 & 54.96 & $\begin{array}{l}\text { Silt } \\
\text { loam }\end{array}$ \\
\hline & & $40-120$ & 8.50 & 106.44 & 0.90 & 178.50 & 87.40 & 49.19 & 57.52 & Clay \\
\hline & \multirow{2}{*}{37} & $0-25$ & 8.30 & 80.73 & 3.10 & 67.60 & 32.50 & 19.52 & 52.85 & $\begin{array}{c}\text { Silt } \\
\text { loam }\end{array}$ \\
\hline & & $25-120$ & 8.50 & 98.13 & 1.10 & 309.30 & 56.70 & 47.27 & 55.31 & Clay \\
\hline
\end{tabular}




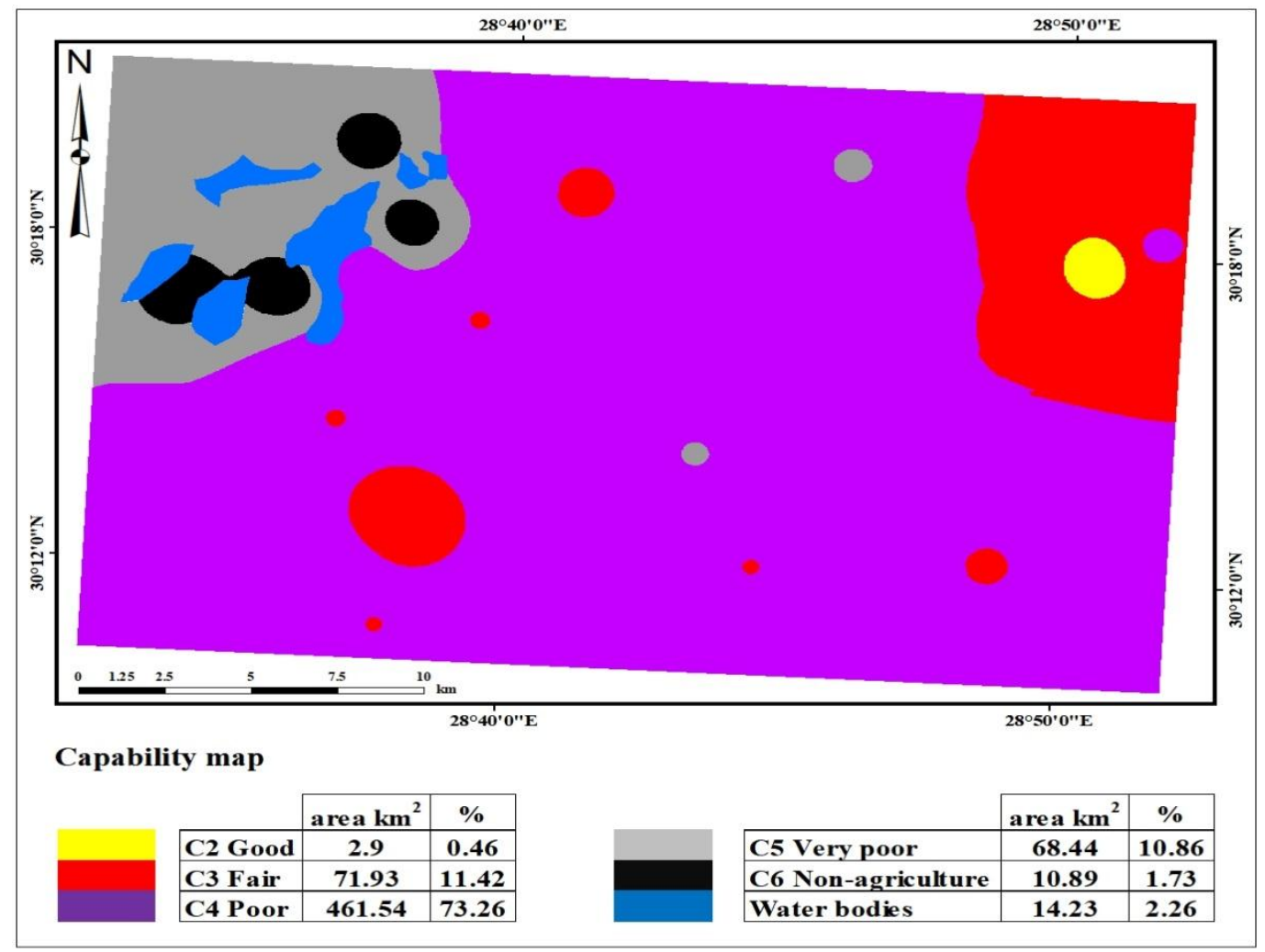

Fig. 4. Land capability map of the studied area

\section{Land suitability classification}

The spatial distribution of suitability classes in the studied area (Table 3 and Fig. 5) indicates that the soils are highly suitable (S1), suitable (S2), moderately suitable (S3), marginally suitable (S4), currently not-suitable (N1) and permanently notsuitable (N2) for the selected crops. Generally, soils belong to the order suitable (S1, S2, S3 and S4) cover more than $90 \%$ of the studied area for all the selected crops, except for faba bean, pea and citrus. For these crops, the soils of the order "suitable" occupy 80.99 , 81.01 and $88.01 \%$ of the total area, respectively.

\section{Conclusion}

Land resources assessment plays a crucial role in land use planning and helps decision makers in sustainable agricultural planning. Such a procedure would be more effective and accurate when integrating modern technologies; RS and GIS with ASLE software. A total area of $630 \mathrm{~km}^{2}$ located in the eastern part of Qattara Depression is classified into 5 capability classes; good (C2), fair (C3), poor (C4), very poor (C5) and non-agricultural (C6). Poor soils occupy the majority of the area $(74.96 \%)$, while the remaining area is classified as"good" $(0.47 \%)$, "fair" $(11.68 \%)$, "very poor" $(11.12 \%)$ and "nonagricultural" $(1.77 \%)$. The main limiting factors are soil texture, salinity and alkalinity. The soils are highly suitable (S1), suitable (S2), moderately suitable (S3), marginally suitable (S4), currently not-suitable (N1) and permanently not-suitable (N2) for the selected 22 crops. The most recommended crops are date palm and tomato.

Table 3. Land suitability classification of the studied area

\begin{tabular}{|c|c|c|c|c|c|c|c|c|c|c|c|c|}
\hline \multirow[t]{2}{*}{ Crop } & \multicolumn{2}{|c|}{$\begin{array}{l}\text { High suitable } \\
\qquad \text { (S1) }\end{array}$} & \multicolumn{2}{|c|}{ Suitable (S2) } & \multicolumn{2}{|c|}{$\begin{array}{l}\text { Moderately } \\
\text { suitable (S3) }\end{array}$} & \multicolumn{2}{|c|}{$\begin{array}{c}\text { Marginally } \\
\text { suitable (S4) }\end{array}$} & \multicolumn{2}{|c|}{$\begin{array}{c}\text { Currently } \\
\text { not suitable } \\
(\mathrm{N} 1) \\
\end{array}$} & \multicolumn{2}{|c|}{$\begin{array}{c}\text { Permanently } \\
\text { not suitable } \\
\text { (N2) }\end{array}$} \\
\hline & $\begin{array}{c}\text { Area, } \\
\mathrm{km}^{2}\end{array}$ & $\begin{array}{c}\text { Area } \\
, \%\end{array}$ & $\begin{array}{c}\text { Area, } \\
\mathrm{km}^{2}\end{array}$ & $\begin{array}{c}\text { Area } \\
, \%\end{array}$ & $\begin{array}{c}\text { Area, } \\
\mathrm{km}^{2}\end{array}$ & $\begin{array}{c}\text { Area } \\
, \%\end{array}$ & $\begin{array}{l}\text { Area, } \\
\mathrm{km}^{2}\end{array}$ & $\begin{array}{c}\text { Area } \\
, \%\end{array}$ & $\begin{array}{c}\text { Area, } \\
\mathrm{km}^{2}\end{array}$ & $\begin{array}{c}\text { Area } \\
, \%\end{array}$ & $\begin{array}{c}\text { Area, } \\
\mathrm{km}^{2}\end{array}$ & $\begin{array}{c}\text { Area } \\
, \%\end{array}$ \\
\hline Alfalfa & 0.76 & 0.12 & 89.42 & $\begin{array}{c}14.5 \\
2\end{array}$ & $\begin{array}{c}398.5 \\
6\end{array}$ & $\begin{array}{c}64.7 \\
3\end{array}$ & 88.04 & $\begin{array}{c}14.3 \\
0\end{array}$ & 31.80 & 5.16 & 7.12 & 1.16 \\
\hline Barley & 0.93 & 0.15 & $\begin{array}{c}119.7 \\
9\end{array}$ & $\begin{array}{c}19.4 \\
6\end{array}$ & $\begin{array}{c}407.5 \\
3\end{array}$ & $\begin{array}{c}66.1 \\
9\end{array}$ & 59.43 & 9.65 & 22.16 & 3.60 & 5.87 & 0.95 \\
\hline $\begin{array}{l}\text { Faba } \\
\text { bean }\end{array}$ & 0.10 & 0.02 & 26.87 & 4.36 & $\begin{array}{c}323.2 \\
8 \\
\end{array}$ & $\begin{array}{c}52.5 \\
1 \\
\end{array}$ & $\begin{array}{c}148.4 \\
0 \\
\end{array}$ & $\begin{array}{c}24.1 \\
0 \\
\end{array}$ & $\begin{array}{c}100.2 \\
3 \\
\end{array}$ & $\begin{array}{c}16.2 \\
8 \\
\end{array}$ & 16.82 & 2.73 \\
\hline
\end{tabular}




\begin{tabular}{|c|c|c|c|c|c|c|c|c|c|c|c|c|}
\hline Maize & 0.79 & 0.13 & 55.87 & 9.07 & $\begin{array}{c}391.5 \\
5\end{array}$ & $\begin{array}{c}63.5 \\
9\end{array}$ & $\begin{array}{c}18.1 \\
3\end{array}$ & $\begin{array}{c}19.1 \\
9\end{array}$ & 39.18 & 6.36 & 10.19 & 1.66 \\
\hline Peanut & 8.04 & 1.31 & $\begin{array}{c}265.5 \\
4\end{array}$ & $\begin{array}{c}43.1 \\
3\end{array}$ & $\begin{array}{c}148.7 \\
5\end{array}$ & $\begin{array}{c}24.1 \\
6\end{array}$ & $\begin{array}{c}129.8 \\
2\end{array}$ & $\begin{array}{c}21.0 \\
8\end{array}$ & 53.19 & 8.64 & 10.36 & 1.68 \\
\hline $\begin{array}{c}\text { Sorghu } \\
\mathrm{m}\end{array}$ & 1.15 & 0.19 & $\begin{array}{c}100.2 \\
1\end{array}$ & $\begin{array}{c}16.2 \\
8\end{array}$ & $\begin{array}{c}371.2 \\
4\end{array}$ & $\begin{array}{c}60.3 \\
0\end{array}$ & $\begin{array}{c}101.0 \\
2\end{array}$ & $\begin{array}{c}16.4 \\
1\end{array}$ & 32.55 & 5.29 & 9.53 & 1.55 \\
\hline $\begin{array}{c}\text { Soybea } \\
n\end{array}$ & 0.10 & 0.02 & 26.87 & 4.36 & $\begin{array}{c}323.2 \\
9\end{array}$ & $\begin{array}{c}52.5 \\
1\end{array}$ & $\begin{array}{c}148.4 \\
9\end{array}$ & $\begin{array}{c}24.1 \\
2\end{array}$ & $\begin{array}{c}100.1 \\
8\end{array}$ & $\begin{array}{c}16.2 \\
7\end{array}$ & 16.78 & 2.73 \\
\hline $\begin{array}{c}\text { Sugar } \\
\text { beet }\end{array}$ & 4.16 & 0.68 & $\begin{array}{c}236.1 \\
7\end{array}$ & $\begin{array}{c}38.3 \\
6\end{array}$ & $\begin{array}{c}292.0 \\
6\end{array}$ & $\begin{array}{c}47.4 \\
4\end{array}$ & 59.58 & 9.68 & 18.53 & 3.01 & 5.19 & 0.84 \\
\hline $\begin{array}{l}\text { Sunflo } \\
\text { wer }\end{array}$ & 4.16 & 0.68 & $\begin{array}{c}236.1 \\
7\end{array}$ & $\begin{array}{c}38.3 \\
6\end{array}$ & $\begin{array}{c}292.0 \\
6\end{array}$ & $\begin{array}{c}47.4 \\
4\end{array}$ & 59.58 & 9.68 & 18.53 & 3.01 & 5.19 & 0.84 \\
\hline Wheat & 0.88 & 0.14 & $\begin{array}{c}111.3 \\
1\end{array}$ & $\begin{array}{c}18.0 \\
8\end{array}$ & $\begin{array}{c}414.9 \\
8\end{array}$ & $\begin{array}{c}67.4 \\
0\end{array}$ & 60.09 & 9.76 & 22.53 & 3.66 & 5.90 & 0.96 \\
\hline $\begin{array}{c}\text { Cabbag } \\
\mathrm{e}\end{array}$ & 2.62 & 0.43 & $\begin{array}{c}170.0 \\
1\end{array}$ & $\begin{array}{c}27.6 \\
1\end{array}$ & $\begin{array}{c}308.9 \\
8\end{array}$ & $\begin{array}{c}50.1 \\
8\end{array}$ & 98.60 & $\begin{array}{c}16.0 \\
1\end{array}$ & 29.23 & 4.75 & 6.26 & 1.02 \\
\hline Onion & 0.92 & 0.15 & 99.00 & $\begin{array}{c}16.0 \\
8\end{array}$ & $\begin{array}{c}313.4 \\
4\end{array}$ & $\begin{array}{c}50.9 \\
1\end{array}$ & $\begin{array}{c}136.1 \\
7\end{array}$ & $\begin{array}{c}22.1 \\
2\end{array}$ & 57.59 & 9.35 & 8.58 & 1.39 \\
\hline Pea & 0.79 & 0.13 & 75.21 & $\begin{array}{c}12.2 \\
2\end{array}$ & $\begin{array}{c}287.7 \\
2\end{array}$ & $\begin{array}{c}46.7 \\
3\end{array}$ & $\begin{array}{c}157.5 \\
7\end{array}$ & $\begin{array}{c}25.5 \\
9\end{array}$ & 83.98 & $\begin{array}{c}13.6 \\
4\end{array}$ & 10.45 & 1.70 \\
\hline Pepper & 13.90 & 2.26 & $\begin{array}{c}290.0 \\
7\end{array}$ & $\begin{array}{c}47.1 \\
1\end{array}$ & $\begin{array}{c}142.5 \\
1\end{array}$ & $\begin{array}{c}23.1 \\
5\end{array}$ & $\begin{array}{c}123.7 \\
5\end{array}$ & $\begin{array}{c}20.1 \\
0\end{array}$ & 39.21 & 6.37 & 6.27 & 1.02 \\
\hline Potato & 6.36 & 1.03 & $\begin{array}{c}268.0 \\
0\end{array}$ & $\begin{array}{c}43.5 \\
3\end{array}$ & $\begin{array}{c}200.4 \\
6\end{array}$ & $\begin{array}{c}32.5 \\
6\end{array}$ & $\begin{array}{c}105.0 \\
8\end{array}$ & $\begin{array}{c}17.0 \\
7\end{array}$ & 27.77 & 4.51 & 8.03 & 1.30 \\
\hline Tomato & 39.68 & 6.44 & $\begin{array}{c}360.0 \\
5\end{array}$ & $\begin{array}{c}58.4 \\
8\end{array}$ & $\begin{array}{c}105.6 \\
0\end{array}$ & $\begin{array}{c}17.1 \\
5\end{array}$ & 87.15 & $\begin{array}{c}14.1 \\
5\end{array}$ & 18.86 & 3.06 & 4.38 & 0.71 \\
\hline $\begin{array}{l}\text { Water } \\
\text { melon }\end{array}$ & 9.71 & 1.58 & $\begin{array}{c}269.6 \\
2\end{array}$ & $\begin{array}{c}43.7 \\
9\end{array}$ & $\begin{array}{c}198.2 \\
0\end{array}$ & $\begin{array}{c}32.1 \\
9\end{array}$ & $\begin{array}{c}104.3 \\
5\end{array}$ & $\begin{array}{c}16.9 \\
5\end{array}$ & 26.14 & 4.25 & 7.67 & 1.25 \\
\hline Citrus & 1.60 & 0.26 & 74.86 & $\begin{array}{c}12.1 \\
6\end{array}$ & $\begin{array}{c}337.0 \\
2\end{array}$ & $\begin{array}{c}54.7 \\
4\end{array}$ & $\begin{array}{c}128.4 \\
2\end{array}$ & $\begin{array}{c}20.8 \\
6\end{array}$ & 63.01 & $\begin{array}{c}10.2 \\
3\end{array}$ & 10.80 & 1.75 \\
\hline $\begin{array}{l}\text { Date } \\
\text { palm }\end{array}$ & 52.63 & 8.55 & $\begin{array}{c}427.2 \\
5\end{array}$ & $\begin{array}{c}69.3 \\
9\end{array}$ & 59.21 & 9.62 & 59.71 & 9.70 & 12.73 & 2.07 & 4.17 & 0.68 \\
\hline Fig & 24.05 & 3.91 & $\begin{array}{c}372.7 \\
8\end{array}$ & $\begin{array}{c}60.5 \\
5\end{array}$ & $\begin{array}{c}106.9 \\
1\end{array}$ & $\begin{array}{c}17.3 \\
6\end{array}$ & 85.86 & $\begin{array}{c}13.9 \\
5\end{array}$ & 20.81 & 3.38 & 5.29 & 0.86 \\
\hline Grape & 13.99 & 2.27 & $\begin{array}{c}297.0 \\
0\end{array}$ & $\begin{array}{c}48.2 \\
4\end{array}$ & $\begin{array}{c}180.8 \\
8\end{array}$ & $\begin{array}{c}29.3 \\
8\end{array}$ & 91.94 & $\begin{array}{c}14.9 \\
3\end{array}$ & 24.28 & 3.94 & 7.60 & 1.23 \\
\hline Olive & 24.05 & 3.91 & $\begin{array}{c}372.7 \\
8\end{array}$ & $\begin{array}{c}60.5 \\
5\end{array}$ & $\begin{array}{c}106.9 \\
1\end{array}$ & $\begin{array}{c}17.3 \\
6\end{array}$ & 85.86 & $\begin{array}{c}13.9 \\
5\end{array}$ & 20.81 & 3.38 & 5.29 & 0.86 \\
\hline
\end{tabular}



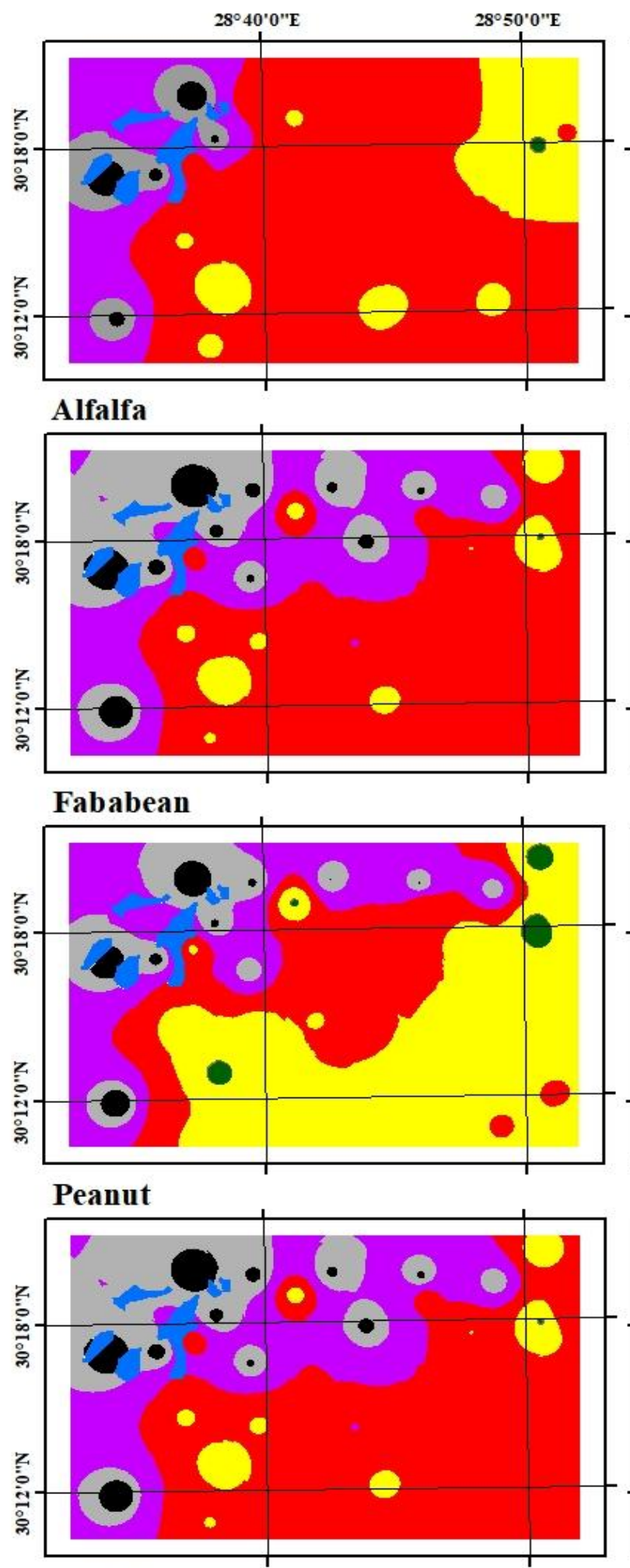

Soya bean

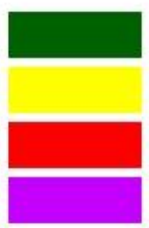

S1 Highly suitable S2 Suitable S3 Moderately suitable S4 Marginally suitable
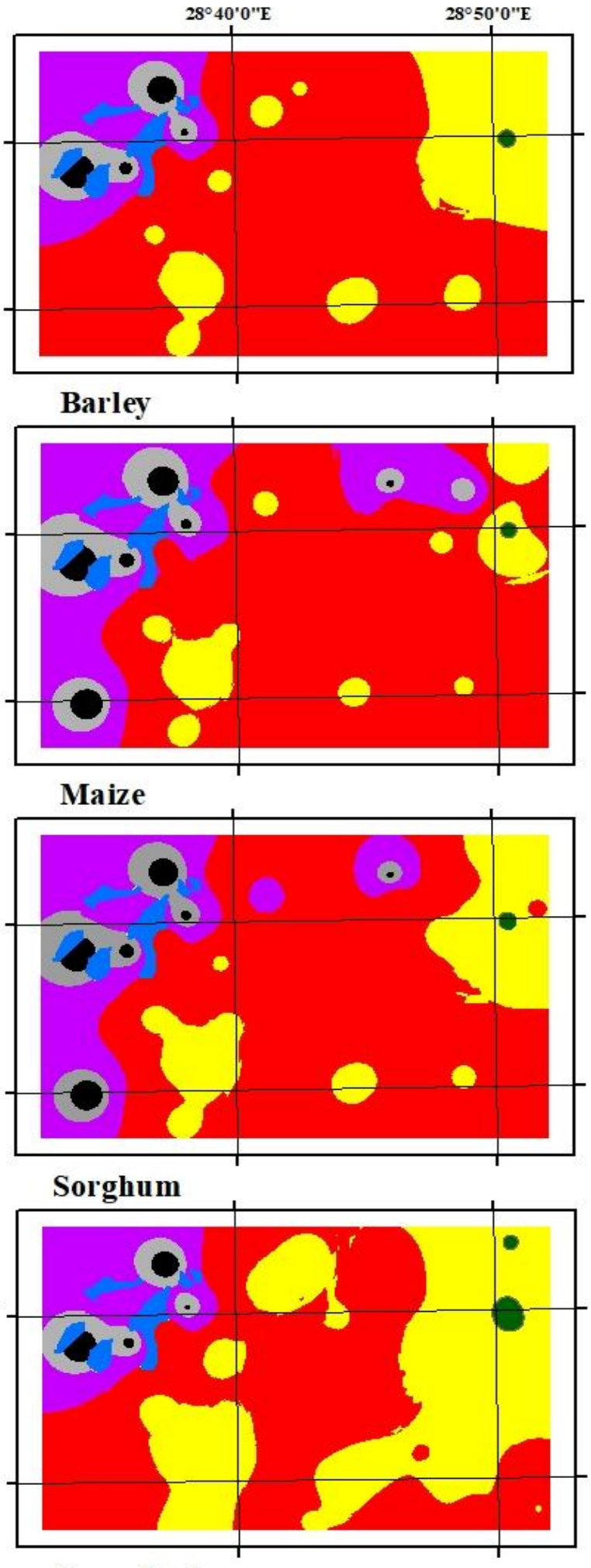

Sugarbeet

Ns1 Currently not suitable

Ns2 Permanently not suitable Water bodies

Fig. 5. Land suitably map of the studied area 

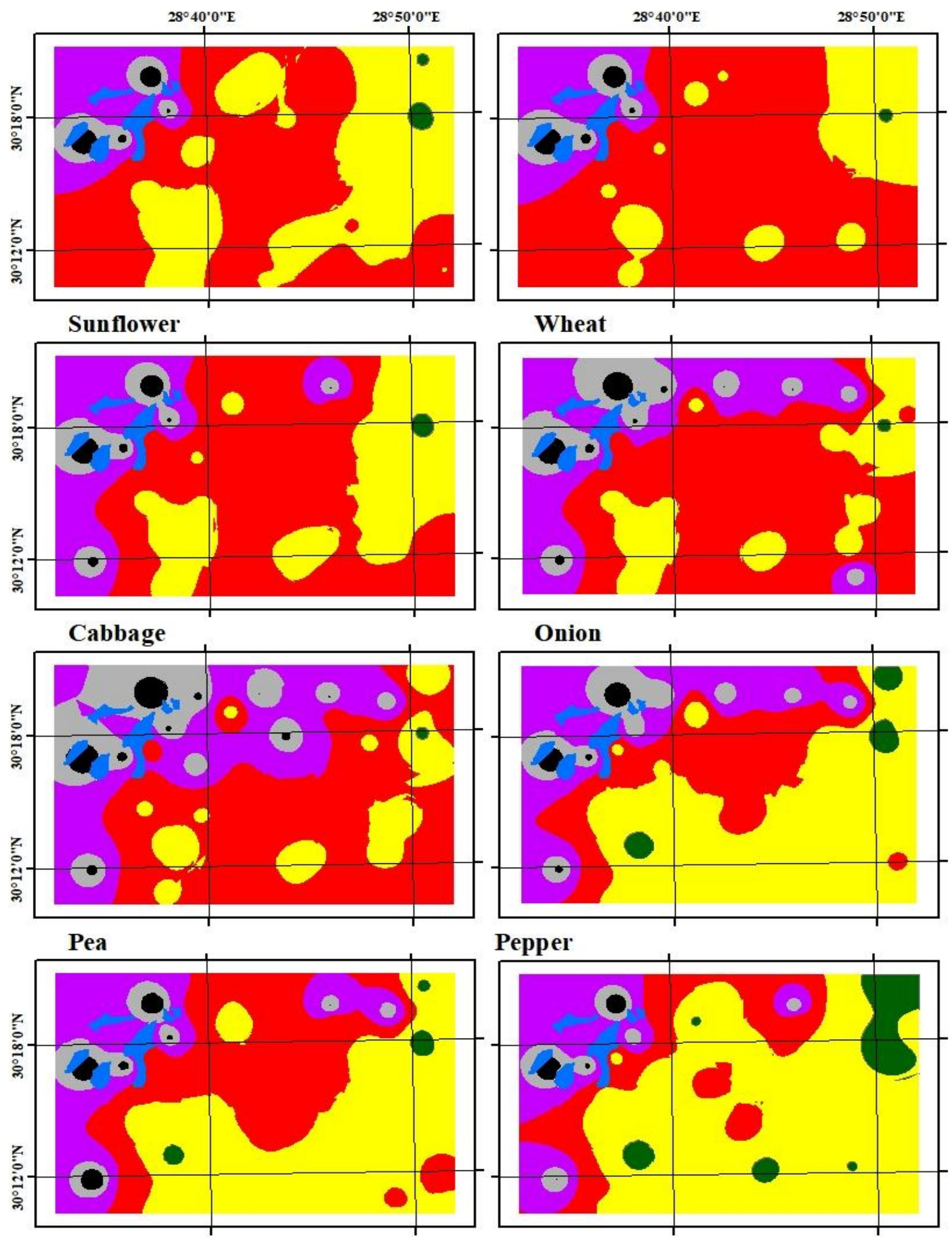

Potato

Tomato

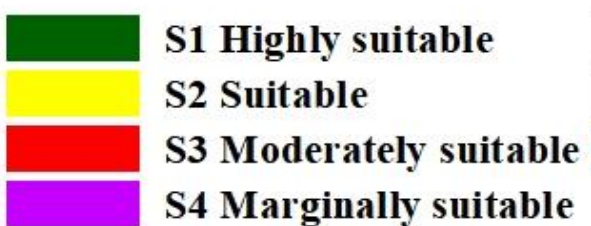

Ns1 Currently not suitable

Ns2 Permanently not suitable Water bodies S4 Marginally suitable

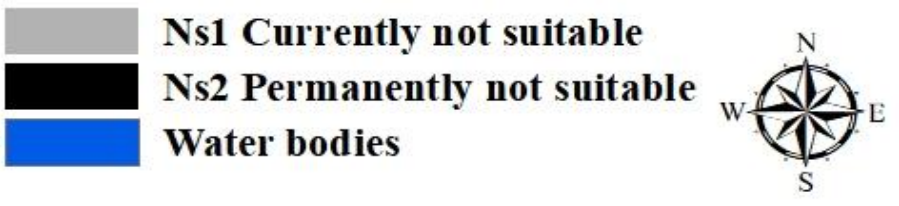

Fig. 5 Cont. 

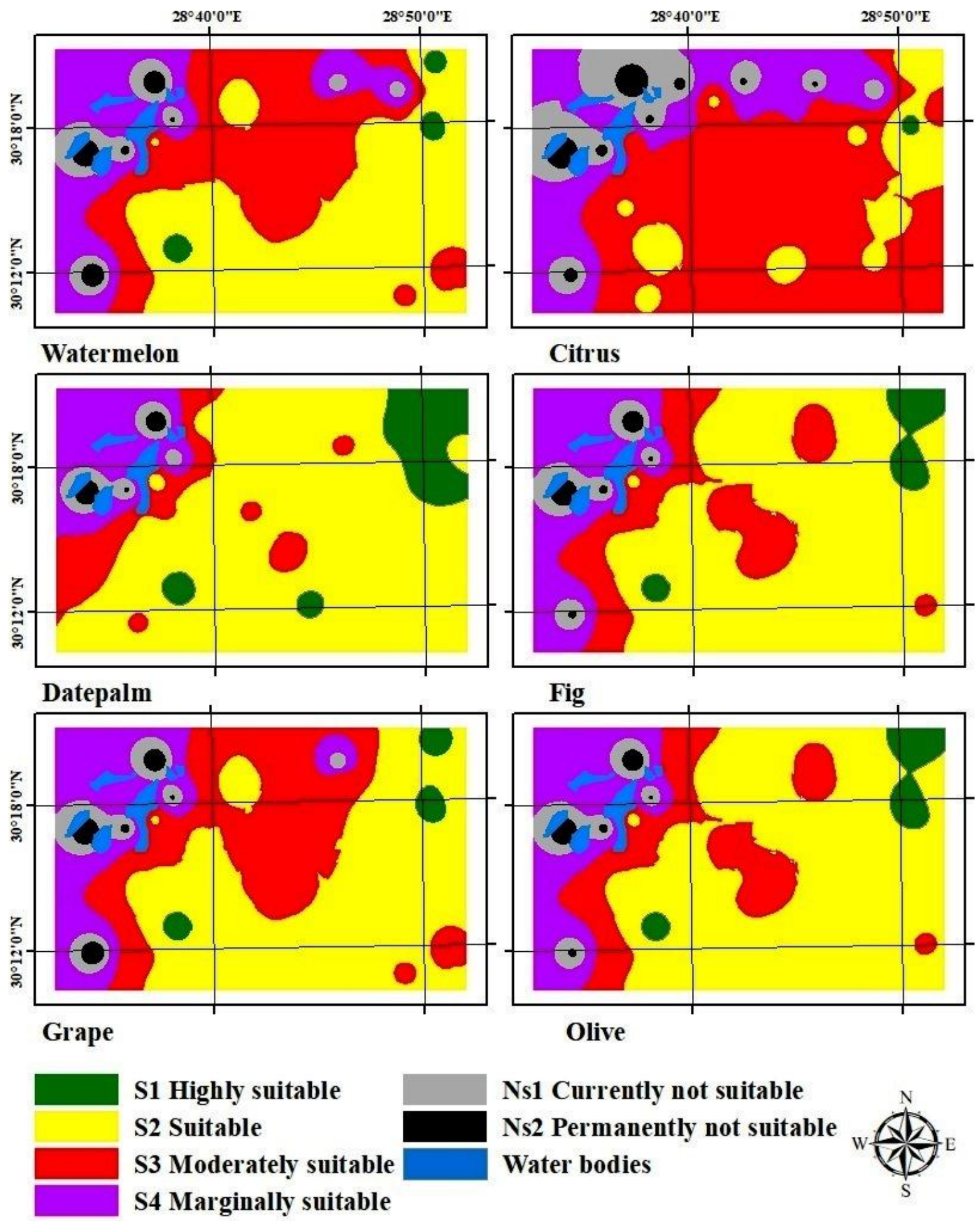

Olive

S1 Highly suitable S2 Suitable S3 Moderately suitable S4 Marginally suitable

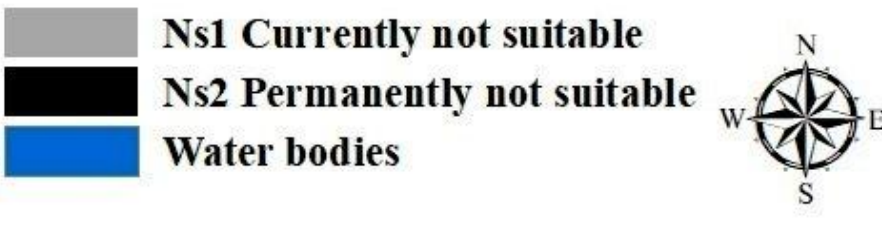

Fig. 5 Cont. 


\section{References}

Abuzaid, A.S., Fadl, M.E. (2016). Land evaluation of eastern Suez Canal, Egypt using remote sensing and GIS. Egypt. J. Soil Sci.56, 537-547.

Bacic, I.L.Z., Pivetta, J.R., Martins, R.P. (2013). Participatory soil and land evaluation mapping: An alternative approach to improve soil and land evaluation information for decision makers. In: Shahid, S.A., Taha, F.K., Abdelfattah, M.A. (Eds.), Developments in soil Classification, land use planning and policy implications: Innovative thinking of soil inventory for land use planning and management of land resources. Springer Netherlands, Dordrecht, pp. 605-615.

Bassouny, M.A., Abuzaid, A.S. (2017). Impact of biogas slurry on some physical properties in sandy and calcareous soils, Egypt. Int. J. Plant Soil Sci16, 1-11.

Embabi, N.S. (2018). The Qattara Depression. In: Embabi, N.S. (Ed.), Landscapes and landforms of Egypt: Landforms and evolution. Springer International Publishing, Cham, pp. 121-132.

FAO (1988). Salt-affected soils and their management. FAO Soils Bulletin No. 39. FAO, Rome, Italy.

FAO (1990). Management of gypsiferous soils. FAO Soils Bulletin 62. Food and Agriculture Organization of the United Nations, Rome, Italy.

FAO (2006). Guidelines for soil description. FAO, Rome, Italy.

Hazelton, P., Murphy, B. (2016). Interpreting soil test results: what do all the numbers mean? CSIRO publishing, Collingwood Victoria, Australia.

Ismail, H.A., Morsy, I.M. (2001). Applied System of Land Evaluation (ASLE) in arid zones (software). Soil and water Science Department, Faculty of Agriculture, Alexandria University, Egypt.

Ismail, M., Yacoub, R.K., Shoman, M.M. (2013). Land suitability evaluation of the eastern part of El-Qattara Depression in Egypt using RS \& GIS Minufiya Journal of Agricultural Research38, 743-1761.

Jafari, M., Tavili, A., Panahi, F., Zandi Esfahan, E., Ghorbani, M. (2018). Amendment of saline and alkaline soils. In: Jafari, M., Tavili, A., Panahi, F., Zandi Esfahan, E., Ghorbani, M. (Eds.), Reclamation of Arid Lands. Springer International Publishing, Cham, pp. 167-174.

Mohamed, E.S., Abu-Hashim, M., Belal, A.-A.A. (2019). Sustainable indicators in arid region: Case study - Egypt. In: Negm, A.M., Abu-hashim, M. (Eds.), Sustainability of agricultural environment in Egypt: Part I: Soil-water-food nexus. Springer International Publishing, Cham, pp. 273-293.

Reddy, G.P.O. (2018a). Geographic Information System: Principles and Applications. In: Reddy, G.P.O., Singh, S.K. (Eds.), Geospatial Technologies in Land Resources Mapping,
Monitoring and Management. Springer International Publishing, Cham, pp. 45-62.

Reddy, G.P.O. (2018b). Geospatial technologies in land resources mapping, monitoring, and management: An overview. In: Reddy, G.P.O., Singh, S.K. (Eds.), Geospatial technologies in land resources mapping, monitoring and management. Springer International Publishing, Cham, pp. 1-18.

Reddy, G.P.O., Ramamurthy, V., Singh, S.K. (2018). Integrated remote sensing, GIS, and GPS applications in agricultural land use planning. In: Reddy, G.P.O., Singh, S.K. (Eds.), Geospatial technologies in land resources mapping, monitoring and management. Springer International Publishing, Cham, pp. 489-515.

Sakai, T. (2012). Remote sensing and geographic information systems. In: Ishida, T. (Ed.), Field informatics: Kyoto University field informatics research group. Springer Berlin Heidelberg, Berlin, Heidelberg, pp. 1-20.

Soil Science Division Staff (2017). Soil survey manual. USDA Handbook 18. Government Printing Office, Washington, D.C, USA.

Soil Survey Staff (2014). Soil survey field and laboratory methods manual. Soil Survey Investigations Report No. 51, Version 2.0. R. Burt and Soil Survey Staff (ed.). U.S. Department of Agriculture, Natural Resources Conservation Service, Washington, DC.

Soliman, S. (2017). Food security and vocational education and training: Exploring the links in the Egyptian case. In: Mergos, G., Papanastassiou, M. (Eds.), Food security and sustainability: Investment and financing along agro-food chains. Springer International Publishing, Cham, pp. 193208.

Sys, C., Van Ranst, E., Debaveye, J., Beernaert, F. (1993). Land evaluation. Part III. Crop requirements. Agricultural publications No. 7. General administration for development cooperation, Brussels.

Vasu, D., Srivastava, R., Patil, N.G., Tiwary, P., Chandran, P., Kumar Singh, S. (2018). A comparative assessment of land suitability evaluation methods for agricultural land use planning at village level. Land Use Policy79, 146163.

Xue, F. (2011). Land evaluation supported by MDS. In: Li, D., Liu, Y., Chen, Y. (Eds.), Computer and computing technologies in agriculture IV. CCTA 2010. IFIP Advances in information and communication technology, vol 347. Springer Berlin Heidelberg, Berlin, Heidelberg, pp. 623628.

Zahran, M.A., Willis, A.J. (2009). The Western Desert. In: Zahran, M.A., Willis, A.J. (Eds.), The Vegetation of Egypt. Springer Netherlands, Dordrecht, pp. 13-100. 
Zinck, J.A., Valenzuela, C.R. (1990). Soil geographic database: structure and application examples. ITC Journal3, 270-294.

\section{تقييم الأراضي بشرق منخفض القطارة - مصر بإستخدام الإستشعار عن بعد ونظم المعلومات الجغرافية

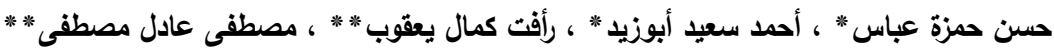

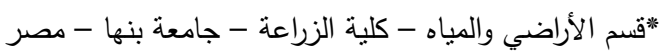

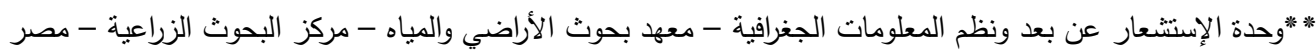

تم إستخدام بيانات القمر الصناعي Sntinel-2Aونظام التحليل الإحصائي الدكاني خلال منظومة المعلومات الجغرافية لإنتاج خرائط قدرة التربة وملاءمنها للزراعة ببعض المحاصيل في مساحة قرها 630 كم2 (63000 هكتار) من الأراضي الواقعة بشرق منخفض القطارة مصر ـ تم تمثيل

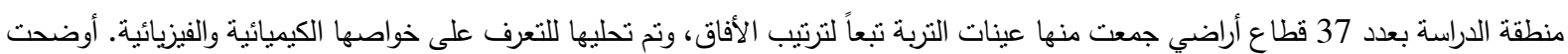

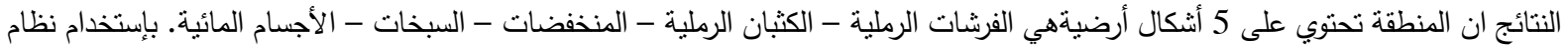

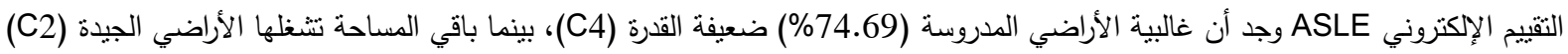

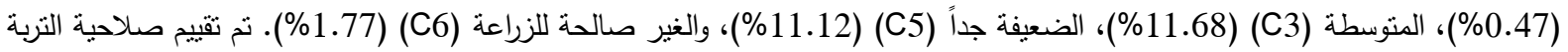

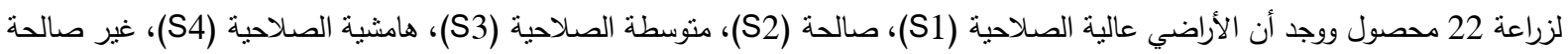
حالياً (N1)، وغير صالحة مستقبلاً (N2) للمحاصيل المختارة. وجد أن أفضل المحاصيل الموصى بزراعتها هي نخيل البلح والطماطر. 